\title{
ACE-2 expression in the small airway epithelia of smokers and COPD patients: implications for COVID-19
}

\author{
To the Editor:
}

The World Health Organization (WHO) has declared coronavirus disease 2019 (COVID-19) a pandemic [1]. COVID-19 is caused by severe acute respiratory syndrome coronavirus 2 (SARS-CoV-2). COVID-19 displays symptoms ranging from mild to severe (pneumonia) that can lead to death in some individuals [2-4]. As of 18 April 2020, there have been 2280945 cases of COVID-19 worldwide and 156354 deaths [5]. SARS-CoV-2 uses the angiotensin-converting enzyme II (ACE-2) as the cellular entry receptor [6]. While the virus can infect individuals of any age, to date, most of the severe cases have been described in those $>55$ years of age and with significant comorbidities, such as COPD [7]. Here, we determined whether patients with COPD have increased expression of ACE-2 in bronchial epithelial cells in the lower respiratory tract.

Patients undergoing bronchoscopy at St Paul's Hospital (Vancouver, BC, Canada) for clinical purposes were enrolled. The protocol was approved by the University of British Columbia/Providence Health Care Ethics Board (UBC/PHC REB H15-02166). All patients were required to be $\geqslant 19$ years of age and undergoing spirometry according to international guidelines [8]. Patients with COPD were defined as those having a clinical diagnosis of COPD made by a board-certified respiratory physician and either a forced expiratory volume in $1 \mathrm{~s}\left(\mathrm{FEV}_{1}\right) /$ forced vital capacity $(\mathrm{FVC})<70 \%$ or clear evidence of emphysema on computed tomography imaging on visual inspection. Cytological brushings were obtained in subsegmental airways (6th-8th generation) of the lung that were unaffected by the patient's underlying clinical indication for bronchoscopy.

Total RNA was extracted from cytological brushings using the RNeasy Mini Kit (Qiagen, Hilden, Germany). Transcriptomic sequencing was performed on the NovaSeq 6000 (Illumina, San Diego, CA, USA) at a sequencing depth of 55 million reads. Raw sequencing reads were quality controlled with FastQC [9] and aligned to the GENCODE (version 31) GRCh37 genome reference using STAR (spliced transcripts alignment to a reference) [10]. After alignment, the data were quantified using RSEM (RNA-seq by expectation maximisation) to obtain the read counts. Limma voom [11] was applied to normalise the counts to $\log 2$ counts per million (CPM) reads, which was used in the downstream analysis.

Two cohorts were used for validation, the details of which are provided in a previous publication [12]. First, we used 16 datasets obtained from bronchial brushings of 10th-12th generation bronchi collected at a single centre; transcriptome measurement was performed using the U133 Plus 2.0 (ThermoFisher, Waltham, MA, USA) microarray (denoted as the Cornell cohort) [13]. Secondly, we used dataset GSE37147 consisting of bronchial brushings from the 6th-8th generation airways with gene expression profiles generated from the GeneChip Human Gene 1.0 ST microarray (ThermoFisher) [14]. This dataset was denoted as the British Columbia Cancer Agency (BCCA) cohort.

We also determined the protein expression of ACE-2 in resected lung tissue specimens. These samples were obtained from 10 current smokers with COPD (mean \pm SD $\mathrm{FEV}_{1} / \mathrm{FVC} 61 \pm 7 \%$ ), nine nonsmoker controls $\left(\mathrm{FEV}_{1} / \mathrm{FVC} 85 \pm 2 \%\right)$ and eight healthy current smokers (FEV $\left.1 / \mathrm{FVC} 78 \pm 6 \%\right)$. Human lung tissue samples were obtained with informed consent from patients undergoing thoracic surgery as part of the

@ERSpublications

Smokers and those with COPD have increased airway expression of ACE-2, which is the entry receptor for the COVID-19 virus. This may explain the increased risk of severe COVID-19 in these subpopulations and highlight the importance of smoking cessation. https://bit.ly/3bC29es

Cite this article as: Leung JM, Yang CX, Tam A, et al. ACE-2 expression in the small airway epithelia of smokers and COPD patients: implications for COVID-19. Eur Respir J 2020; 55: 2000688 [https://doi.org/ 10.1183/13993003.00688-2020]. 
James Hogg Lung Registry (UBC/PHC REB Protocol H00-50110). Formalin-fixed paraffin-embedded human lung tissues were stained with antibody against ACE-2 (Ab15348; Abcam, Cambridge, UK) using the Bond Polymer Refine Red Detection kit on a Leica Bond Autostainer (both Leica Biosystems, Concord, ON, Canada), as previously described [15]. Airway epithelial-specific ACE-2 protein intensity was quantified using the Aperio imaging system (Leica Biosystem) with normalisation to the length of the basement membrane.

For the primary study population, $\log 2 \mathrm{CPM}$ of ACE-2 was the principal outcome of interest. Robust linear models were used to determine: 1) whether ACE-2 was differentially expressed in patients with COPD and in smokers after adjustment for age and sex; and 2) whether ACE-2 expression was significantly correlated with lung function. All analyses were performed in R (version 3.5.0; https://cran.rproject.org). In the immunohistochemistry dataset, Kruskal-Wallis with Dunn's multiple comparisons tests was used. Continuous data are expressed as mean $\pm \mathrm{SD}$, unless otherwise indicated.

The average age of the St Paul's Hospital cohort was $64.8 \pm 12.0$ years; $55 \%$ were females and $24 \%$ were current smokers. Compared with control subjects $(n=21)$, those with COPD $(n=21)$ had a lower $\mathrm{FEV}_{1} \%$ predicted (72.0 \pm 15.6 versus $85.9 \pm 17.9 \%$ pred; $\mathrm{p}=0.011)$ and $\mathrm{FEV}_{1} / \mathrm{FVC}(64.1 \pm 7.9$ versus $76.3 \pm 5.9 \%$; $\left.2.621 \times 10^{-6}\right)$. Most $(79 \%)$ underwent bronchoscopy for investigation of lung nodules, followed by chronic cough (7\%) and lymphadenopathy (7\%). ACE-2 expression in the epithelial cells was significantly increased in COPD versus non-COPD subjects (COPD $2.52 \pm 0.66$ versus non-COPD $1.70 \pm 0.51$; $\mathrm{p}=7.62 \times 10^{-4}$; figure 1a). There was a significant inverse relationship between ACE-2 gene expression and $\mathrm{FEV}_{1} \%$ pred $(\mathrm{r}=-0.24 ; \mathrm{p}=0.035$; figure $1 \mathrm{~b})$. Interestingly, smoking status was also significantly related to ACE-2 gene expression levels in the airways of these participants, with current smokers having a significantly higher gene expression than never-smokers (current smokers $2.77 \pm 0.91$ versus never-smokers $1.78 \pm 0.39 ; \mathrm{p}=0.024)$. Former smokers had gene expression levels inbetween that of never- and current smokers (former smokers 2.00土1.23; figure 1c). Adjusted for smoking status, the association between ACE-2 expression and COPD was still significant (adjusted mean \pm SE of non-COPD $0.90 \pm 0.65$ versus COPD 1.75 $\pm 0.82 ; \mathrm{p}=0.016)$.

Next, we validated the above findings in: 1$)$ the Cornell cohort $(n=211)$; and 2) the BCCA cohort $(n=238)$. The average age of the Cornell cohort was $43.6 \pm 10.5$ years; $33.2 \%$ of the cohort were female. $32.2 \%$ were never-smokers and $67.8 \%$ were current smokers at the time of bronchoscopy. The average age of the BCCA cohort was $64.5 \pm 5.9$ years; $43.3 \%$ of the cohort were female. All were heavy smokers with $\geqslant 30$ pack-years of smoking. Of these, $41.6 \%$ were current smokers at the time of bronchoscopy and the remaining were former smokers.

In both the Cornell and BCCA cohorts, current smokers had increased ACE-2 gene expression levels in the airways compared with never-smokers (in the Cornell cohort, current smokers $4.34 \pm 0.45$ versus never smokers $4.15 \pm 0.36 ; \mathrm{p}=1.92 \times 10^{-3}$ ) and former smokers (in the BCCA cohort, current smokers $6.05 \pm 0.53$ versus former smokers $\left.5.57 \pm 0.37 ; \mathrm{p}<2 \times 10^{-16}\right)$. In the $\mathrm{BCCA}$ cohort, pre-bronchodilator $\mathrm{FEV}_{1}$ was measured and was significantly related to ACE-2 gene expression level $(r=-0.10 ; p=0.037)$.

Representative images of epithelial-specific ACE-2 protein expression in nonsmokers, healthy smokers and smokers with COPD are shown in figure 1d. ACE-2 expression in the human small airway epithelium was significantly increased in COPD compared with nonsmokers, but not in healthy smokers (figure 1d). ACE-2 protein staining was largely restricted to the airway epithelium and cells in the submucosal compartment.

There is a worldwide outbreak of COVID-19. Although most patients infected and diagnosed with COVID-19 have mild symptoms, $20 \%$ of individuals have demonstrated severe or critically severe disease, including symptoms and signs of pneumonia, respiratory failure, septic shock and multi-organ failure. The estimated case-fatality rate is $1-2 \%[2,3]$. Importantly, nearly all deaths have occurred in those with significant underlying chronic diseases, including COPD and cardiovascular diseases [4]. The reason for this is largely unknown.

One possibility is the differential expression of ACE-2, which is the main receptor used by SARS-CoV-2 to gain entry into the host mucosa and cause active infection. In this study, we investigated gene expression levels of ACE-2 in the airways of individuals with and without COPD and found that COPD and current smokers had significantly increased expression of ACE-2. Importantly, gene expression levels of ACE-2 were inversely related to an individual's $\mathrm{FEV}_{1}$, suggesting a dose-dependent response. These findings were observed in three different cohorts, indicating their generalisability and robustness.

ACE-2 is a type I transmembrane metallocarboxypeptidase with homology to ACE. In contrast to ACE, which converts angiotensin I to the active vasoconstrictor, angiotensin II, ACE-2 breaks down angiotensin II to its metabolites, including angiotensin-(1-9) and angiotensin-(1-7), which are potent vasodilators, 

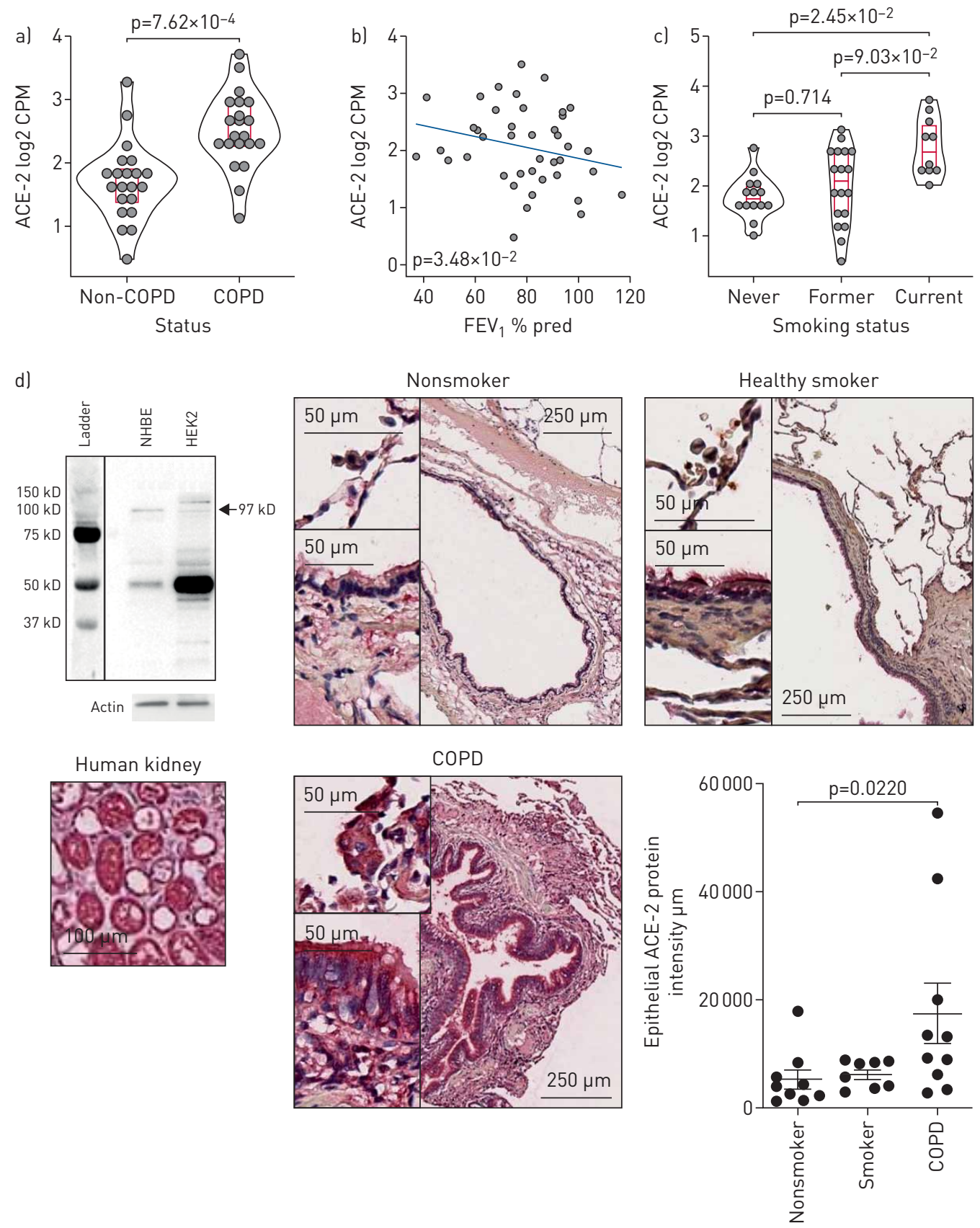

FIGURE 1 a) A violin plot of angiotensin-converting enzyme II (ACE-2) expression the in small airways of COPD and non-COPD subjects in the St Paul's Hospital (Vancouver, BC, Canada) cohort. The red box indicates the median and interquartile range. The $p$-value was obtained using a robust linear model. b) A scatterplot of ACE-2 expression in the small airways according to forced expiratory volume in $1 \mathrm{~s}\left(\mathrm{FEV}_{1}\right) \%$ predicted in the St Paul's Hospital cohort. ACE-2 gene expression in airway epithelia is inversely related to $F E V_{1} \%$ pred ( $p=0.0348$ ). c) A violin plot of ACE-2 expression in the small airways of never-, former and current smokers in the St Paul's Hospital cohort. The red box indicates the median and the interquartile range. The $p$-value was obtained using a robust linear model. d) Protein staining of ACE-2 in the airways of individuals with and without COPD. A human kidney slide was the positive control for ACE-2. The specificity of the antibody against ACE-2 was determined using an immunoblot assay with HEK2 cell lysates as a positive control. The expected molecular weight of ACE-2 is $90-100 \mathrm{kDa}$. In the airways, most of the protein expression was noted in the epithelial layer, and most pronounced in those with COPD. CPM: counts per million; NHBE: normal human bronchial epithelial cells.

and thus may be a negative regulator of the renin-angiotensin system [16]. ACE-2 is expressed in a variety of different tissues, including both the upper and lower respiratory tract, myocardium and the gastrointestinal mucosa [17]. Although its role in human health and disease has not been fully elucidated, it appears to have an important regulatory role in blood pressure and cardiac function. The physiological 
role of ACE-2 in the airways is largely unknown. However, in mice, ACE-2 has been shown to protect animals from severe lung injury related to aspiration and sepsis [18].

To our knowledge, our study is the first to demonstrate increased ACE-2 expression in the airways of current (but not former) smokers and those with COPD. These results are also consistent with previous observations in small animals wherein smoke exposure has been shown to upregulate both the expression and activity of ACE-2 in the airways $[19,20]$. While the upregulation of ACE-2 may be useful in protecting the host against acute lung injury, chronically, this may predispose individuals to an increased risk of coronavirus infections, which use this receptor to gain entrance into epithelial cells. This may in part explain the increased risk of viral respiratory tract infection in active smokers and virus-related exacerbations in those with COPD.

There were limitations to the study. First, the study was cross-sectional and as such, we could not determine whether interventions such as inhaled corticosteroids or bronchodilators (for those with COPD) could modulate ACE-2 gene expression in the airways. Second, as receptor expression is one of many host factors that govern infection risk among individuals, the precise attributable risk (for coronavirus infections) imposed by cigarette smoking and COPD is uncertain. Third, although the airway epithelia are the major source of entry for COVID-19, the virus can gain host entry through other ports, including gastrointestinal mucosa, which was not evaluated in this study. Fourth, we did not have access to upper airway tissues, which may also become infected with SARS-CoV-2.

In summary, active cigarette smoking and COPD upregulate ACE-2 expression in the lower airways, which in part may explain the increased risk of severe COVID-19 in these populations. These findings highlight the importance of smoking cessation for these individuals and increased surveillance of these risk subgroups for prevention and rapid diagnosis of this potentially deadly disease.

Janice M. Leung ${ }^{1,2,3}$, Chen X. Yang ${ }^{1,2,3}$, Anthony Tam $^{1,2,3}$, Tawimas Shaipanich ${ }^{2,3}$, Tillie-Louise Hackett ${ }^{2,3,4}$, Gurpreet K. Singhera ${ }^{1,2,3}$, Delbert R. Dorscheid ${ }^{1,2,3}$ and Don D. $\operatorname{Sin}^{1,2,3}$

${ }^{1}$ University of British Columbia (UBC) Centre for Heart Lung Innovation, Vancouver, BC, Canada. ${ }^{2}$ Dept of Medicine (Division of Respirology), University of British Columbia, Vancouver, BC, Canada. ${ }^{3}$ St Paul's Hospital, Providence Health Care, Vancouver, BC, Canada. ${ }^{4}$ Dept of Anesthesia, Pharmacology and Therapeutics, University of British Columbia, Vancouver, BC, Canada.

Correspondence: Don D. Sin, UBC Centre for Heart Lung Innovation, St Paul's Hospital, 1081 Burrard Street, Vancouver, BC V6Z1Y6, Canada. E-mail: don.sin@hli.ubc.ca

Received: 2 March 2020 | Accepted after revision: 25 March 2020

Data availability: Raw data available from the authors upon request. The data are also posted on GEO (Gene Expression Omnibus).

Conflict of interest: J.M. Leung has nothing to disclose. C.X. Yang has nothing to disclose. A. Tam has nothing to disclose. T. Shaipanich has nothing to disclose. T.L. Hackett has nothing to disclose. G.K. Singhera has nothing to disclose. D.R. Dorschied has nothing to disclose. D.D. Sin reports grants from Merck, personal fees for advisory board work from Sanofi-Aventis and Regeneron, grants and personal fees for research from Boehringer Ingelheim, grants and personal fees for advisory board work and lectures from AstraZeneca, personal fees for advisory board work and lectures from Novartis, outside the submitted work.

Support statement: This work is supported by the Canadian Institutes of Health Research, the British Columbia Lung Association, and de Lazzari Family Chair at HLI. J.M. Leung is supported by the Michael Smith Foundation for Health Research (MSFHR)/Providence Health Care Health Professional Investigator (HPI) Award and by the Canadian Institutes of Health Research (CIHR)/AstraZeneca Early Career Investigator Award. T-L. Hackett is supported by the MSFHR scholar and CIHR Early Career Investigator awards. D. Dorscheid is supported by the MSFHR/PHC HPI Award. D.D. Sin is a Tier 1 Canada Research Chair (CRC) in COPD and the de Lazzari Family Chair at HLI. Funding information for this article has been deposited with the Crossref Funder Registry.

\section{References}

1 World Health Organization. Coronavirus disease (COVID-19) outbreak. https://www.who.int/emergencies/ diseases/novel-coronavirus-2019/events-as-they-happen Date last accessed: April 18, 2020.

2 Guan WJ, Ni ZY, Hu Y, et al. Clinical characteristics of coronavirus disease 2019 in China. N Engl J Med 2020; 18: 708-720.

3 Li Q, Guan X, Wu P, et al. Early transmission dynamics in Wuhan, China, of novel coronavirus-infected pneumonia. N Engl J Med 2020; 382: 1199-1207.

4 Wu Z, McGoogan JM. Characteristics of and important lessons from the coronavirus disease 2019 (COVID-19) outbreak in China: summary of a report of 72314 cases from the Chinese Center for Disease Control and Prevention. JAMA 2020; in press [https://doi.org/10.1001/jama.2020.2648].

5 Coronavirus Worldometer. https://www.worldometers.info/coronavirus/ Date last accessed: April 18, 2020.

6 Zhou P, Yang XL, Wang XG, et al. A pneumonia outbreak associated with a new coronavirus of probable bat origin. Nature 2020; 579: 270-273. 
7 Wang $\mathrm{D}, \mathrm{Hu} \mathrm{B}, \mathrm{Hu} \mathrm{C}$, et al. Clinical characteristics of 138 hospitalized patients with 2019 nove coronavirus-infected pneumonia in Wuhan, China. JAMA 2020; in press [https://doi.org/10.1001/jama.2020.1585].

8 Graham BL, Steenbruggen I, Miller MR, et al. Standardization of spirometry 2019 update. An official American Thoracic Society and European Respiratory Society technical statement. Am J Respir Crit Care Med 2019; 200: e70-e88.

9 Andrews S. (2010). FastQC: a quality control tool for high throughput sequence data. http://www.bioinformatics. babraham.ac.uk/projects/fastqc/ Date last accessed: April 18, 2020.

10 Dobin A, Davis CA, Schlesinger F, et al. STAR: ultrafast universal RNA-seq aligner. Bioinformatics 2013; 29: $15-21$.

11 Law CW, Chen Y, Shi W, et al. voom: precision weights unlock linear model analysis tools for RNA-seq read counts. Genome Biol 2014; 15: R29.

12 Yang CX, Shi H, Ding I, et al. Widespread sexual dimorphism in the transcriptome of human airway epithelium in response to smoking. Sci Rep 2019; 9: 17600.

13 Strulovici-Barel Y, Omberg L, O'Mahony M, et al. Threshold of biologic responses of the small airway epithelium to low levels of tobacco smoke. Am J Respir Crit Care Med 2010; 182: 1524-1532.

14 Steiling $\mathrm{K}$, van den Berge M, Hijazi K, et al. A dynamic bronchial airway gene expression signature of chronic obstructive pulmonary disease and lung function impairment. Am J Respir Crit Care Med 2013; 187: 933-942.

15 Tam A, Hughes M, McNagny KM, et al. Hedgehog signaling in the airway epithelium of patients with chronic obstructive pulmonary disease. Sci Rep 2019; 9: 3353.

16 Crackower MA, Sarao R, Oudit GY, et al. Angiotensin-converting enzyme 2 is an essential regulator of heart function. Nature 2002; 417: 822-828.

17 Harmer D, Gilbert M, Borman R, et al. Quantitative mRNA expression profiling of ACE 2, a novel homologue of angiotensin converting enzyme. FEBS Lett 2002; 532: 107-110.

18 Imai $\mathrm{Y}, \mathrm{Kuba} \mathrm{K}$, Rao S, et al. Angiotensin-converting enzyme 2 protects from severe acute lung failure. Nature 2005; 436: 112-116.

19 Hung YH, Hsieh WY, Hsieh JS, et al. Alternative roles of STAT3 and MAPK signaling pathways in the MMPs activation and progression of lung injury induced by cigarette smoke exposure in ACE2 knockout mice. Int J Biol Sci 2016; 12: 454-465.

20 Yilin Z, Yandong N, Faguang J. Role of angiotensin-converting enzyme (ACE) and ACE2 in a rat model of smoke inhalation induced acute respiratory distress syndrome. Burns 2015; 41: 1468-1477. 